\title{
Traugott Leberecht Pochmann und seine Bildnisse mit der verdreht abgewinkelten Hand
}

\author{
Harald Marx
}

1 Neuer Nekrolog der Deutschen. Achter Jahrgang, 1830. Erster Theil. Ilmenau 1832, Artikel August [!] Lebrecht Pochmann, S. 360-371; S. 367, 368.

2 Johann Georg Meusel: Etwas über die öffentliche Ausstellung der Churfürstl. Sächs. Academie der Künste in Dresden am 5. März 1806, in: Archiv für Künstler und Kunstfreunde, Zweyten Bandes erstes Heft, Dresden 1807, S. 93.

3 Versteigerungs-Katalog des Auktionshauses Satow, 13. Februar 2010, Lot 99, Abb. S. 26: Bildnis eines sitzenden Mannes. Um oder bald nach 1800 ; Signiert auf der Rückseite; Öl auf Leinwand, $113 \times 95 \mathrm{~cm}$.
Traugott Leberecht Pochmann: Bildnis eines sitzenden Mannes, um oder bald nach 1800 ,

Öl auf Leinwand, $113 \times 95 \mathrm{~cm}$, versteigert am 13. Februar $2010 \mathrm{im}$ Auktionshaus Satow (Deutschland), gegenwärtiger Aufbewahrungsort unbekannt
Der Dresdner Historien- und Bildnismaler Traugott Leberecht Pochmann (1762-1830) war zeitlebens ein Verehrer des flämischen Malers Anton van Dyck (1599-1641). In einem Nachruf, der zwei Jahre nach seinem Tod erschienen ist und dessen Verfasser viele Begebenheiten aus Pochmanns Leben ausführlich erzählt, heißt es: „Eben so offen und wahr benahm er sich in seinem Urtheile über die Kunstleistungen anderer Meister und seine eigenen. Besonders hoch verehrte er Vandyk und äußerte oftmals, daß er Vandyk gewesen zu sein wünsche. Als daher einer seiner Zeitgenossen, der für seine eigenen Leistungen sehr eingenommen war, über ein Gemälde desselben mit tadelndem Tone äußerte: ich würde es nicht so gemacht haben! erwiderte P. ganz ruhig: ,Sie haben vollkommen recht, auch ich würde es nicht so gemacht haben, denn wenn Sie und ich und alle Maler Dresdens in Eins verschmolzen würden, so würden wir noch nicht den zehnten Theil des Werthes der Leistungen Vandyks produziren können."“

Dieser Nachruf ist verfasst worden von „einem ehemaligen Schüler und großen Verehrer des Verewigten, im Herzogthum Sachsen“, der sich aller-

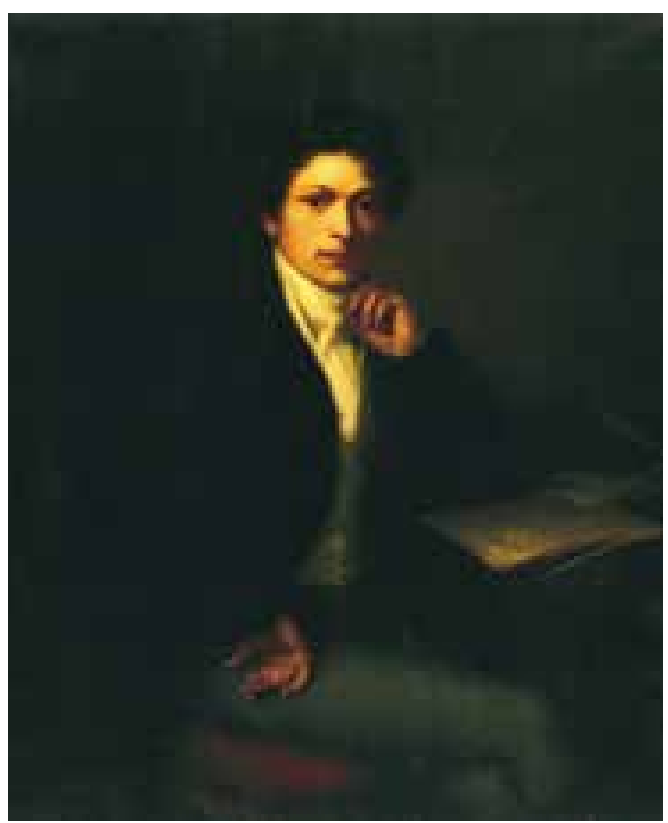

dings nicht namentlich zu erkennen gegeben hat; im Hinblick auf Van Dyck ist der Text deutlich und lässt keinen Zweifel an Pochmanns Verehrung für diesen Maler aus dem 17. Jahrhundert. Die Kunstkenner und Ausstellungs-Rezensenten haben Pochmanns Schaffen während dessen ganzen Lebens stets aufmerksam begleitet und dabei manchmal Lob mit Kritik verbunden; ganz unumstritten war er nie. Beispielsweise riet ihm Johann Georg Meusel 1807 im Archiv für Künstler und Kunstfreunde, nachdem er vorher bei Besprechung des Bildes „Diana und Endymion“, das 1806 ausgestellt worden war, sowohl Lob als auch Einwände vorgebracht hatte, „vorzüglich die Vorbilder zu benutzen, die uns Titian, van Dyck und andere, von der richtigen Vereinigung der Formen der Antike mit den Farben der Natur hinterlassen haben.“2

Wenn wir uns der Begeisterung Pochmanns für Van Dyck erinnern, die ihm im Nachruf bescheinigt wurde, dann nimmt es Wunder, ihn durch den Kritiker 1807 gerade auf dieses Vorbild hingewiesen zu sehen; doch dabei ging es anscheinend um die Verbindung des Studiums nach antiker Skulptur im Gipssaal „mit den Farben der Natur“, also um die Verlebendigung der nach Gipsabgüssen erarbeiteten jeweiligen Komposition. Andererseits wurde bei Bildnissen mehrfach gerade seine gelungene „Carnation" gelobt, also die überzeugende Wiedergabe der Fleischtöne.

Wie sehr sich Pochmann bis in manche Einzelheiten auch die Bildnisse des großen flämischen Malers Anton van Dyck zum Vorbild genommen hat, das sei hier an einem Beispiel erläutert. Es zeigt nämlich, wie gefährlich es sein kann, großen Vorbildern nachfolgen zu wollen, wenn man eigentlich weiß, dass man deren Qualität nicht erreichen kann: Es geht im Folgenden um Bildnisse mit der verdreht abgewinkelten und nachlässig in die Hüfte gestützten Hand.

Hingewiesen sei zuerst auf das „Bildnis eines sitzenden Mannes“, das am 13. Februar 2010 im Auktionshaus Satow versteigert worden ist ${ }^{3}$ es stellt einen eleganten jungen Mann dar, der sehr aufrecht mit übereinander geschlagenen Beinen 
auf einem Stuhl mit rot bezogenem Polster sitzt, nach rechts seitlich gewendet an einem Tisch im nur angedeuteten Innenraum. Vor ihm liegen verschiedene Papiere, denen anscheinend eben noch seine Aufmerksamkeit gegolten hatte. Jetzt aber wendet er sich dem Betrachter zu, schaut ihn an, ohne eigentlich von ihm Notiz zu nehmen. Eine Hand hat er nachdenklich zum Kinn geführt, während die andere seitlich nach außen gedreht auf seinem rechten Oberschenkel ruht: Eine elegant gemeinte, von Van Dyck übernommene und nicht sehr glücklich, sondern etwas künstlich wirkende Geste, die Pochmann mehrfach verwendet hat.

Der gut aussehende und vergeistigt wirkende Dargestellte auf dem eben angesprochenen Bild, in dem man einen Poeten vermuten möchte oder zumindest jemanden mit weit reichenden geistigen Interessen, trägt enge Kniehosen, Hemd, Halsbinde und helle Weste, darüber einen dunklen Rock - ganz der Mode in napoleonischer Zeit entsprechend. Das dunkle, lockige Haar rahmt ein verträumt wirkendes Gesicht. Man möchte die Erfahrungen von Pochmanns Frankreich-Reise der Jahre 1801/02 voraussetzen, um ein solches Bild zu erklären.

Die besondere Haltung der in die Hüfte gestützten und dabei mit der Hand-Innenfläche nach außen gedrehten, leicht abgewinkelten Hand, die der Verfasser auf Bilder von Anton van Dyck als Vorbild zurückführen möchte, kommt bei dem flämischen Maler nicht nur in Bildnissen vor, sondern auch auf Historienbildern, so auf dem Gemälde „Der heilige Ambrosius und Kaiser Theodosius“; von diesem großen Werk haben sich Exemplare im Kunsthistorischen Museum in Wien und in der National Gallery in London erhalten. ${ }^{4}$

Die Komposition dieses Bildes wird auf Peter Paul Rubens zurückgeführt, dem zeitweise und nicht ohne Grund auch das Bildnis eines Mannes in der Dresdner Gemäldegalerie zugeschrieben war, das zwar genau die beschriebene Haltung der Hand zeit, aber so, dass die Hand-Innenfläche nicht sichtbar wird. ${ }^{5}$ Genau dem Typus der Hand jedoch, wie ihn Pochmann verwendet hat, begegnen wir auf anderen Werken von Van Dyck.

Denn der flämische Maler hat Bildnisse mit dieser nachlässig-elegant gemeinten Geste der abgewinkelt mit dem Hand-Rücken in die Hüfte gestützten Hand in unterschiedlichen Varianten später immer wieder geschaffen - und schon bei Rubens finden sich wie erwähnt Werke, die angeführt werden könnten.

Hingewiesen sei hier nur noch auf das große Bildnis von König Karl I. (1600-1649) in Staatsrobe, das sich in der Royal Collection in London befindet ${ }^{6}$ und auf das geradezu aufreizend arrogante Doppelbildnis von Lord John Stuart (1621-1644) und Lord Bernard Stuart, späterer
Earl of Lichfield (1622-1645) in der National Gallery in London; bei letzterem Bild gipfelt die ganze Haltung der Figur in der auf den Oberschenkel gestützten, verdrehten Haltung der behandschuhten Hand.?

Das eigentliche Vorbild für Pochmann dürfte jedoch das jugendliche „Selbstbildnis“ des Anton van Dyck in der Ermitage in St. Petersburg gewesen sein, das sich seit 1772 in der Sammlung von Katharina II. befand - und das durch den Stich von Jan van der Bruggen (geb. 1648/49?ca. 1699) aus dem Jahre 1682 bekannt war. ${ }^{8}$ Dieses Selbstbildnis von Van Dyck hat eine Strahlkraft entwickelt und ist in einer Weise vorbildhaft für spätere Künstler gewesen, die kaum überschätzt werden kann. ${ }^{9}$

Van Dyck hat die in seinen Werken elegant und beinahe wie selbstverständlich wirkende Haltung der verdreht in die Hüfte gestützten Hand später noch mehrfach verwendet, doch dürfte es gerade das heute St. Petersburger Selbstbildnis gewesen sein, das Pochmann verleitet hat, es dem großen flämischen Maler nachtun zu wollen. ${ }^{10}$ An einem solchen Beispiel zeigt sich jedoch, welche Virtuosität dazu gehörte, die etwas gezierte Pose glaubhaft erscheinen $\mathrm{zu}$ lassen - und der Dresdner Maler irrte nicht, wenn er im Gespräch mit einem Künstler bemerkt hatte: „wenn Sie und ich und alle Maler Dresdens in Eins verschmolzen würden, so würden wir noch nicht den zehnten Theil des Werthes der Leistungen Vandyks produziren können."11

Einen offensichtlichen Unterschied allerdings gibt es zu dem Selbstbildnis von Van Dyck, der diese verdrehte Handhaltung bei einer stehenden Figur angewendet hat: Pochmann jedoch zeigt, soweit bisher ersichtlich, nur Sitzfiguren in dieser Haltung. Dadurch wirkt die Hand bei ihm noch mehr geknickt und damit weniger entspannt.

In diesem Zusammenhang sei darauf hingewiesen, dass Pochmann seit seiner Jugend Kupferstiche gesammelt hat; sie machten einen großen Teil des Nachlasses aus; und wenn man sich die Frage stellt, wen von den älteren oder für ihn zeitgenössischen Künstlern Pochmann kannte, ja vielleicht sogar verehrte, so wird man darauf aufmerksam, dass sich in seinem Nachlass, der 1857 in Leipzig versteigert worden ist, viele Künstlerporträts befanden. ${ }^{12}$ Ein Selbstbildnis von Van Dyck war nicht unter den Stichen, die 1857 versteigert worden sind; dafür mag es Gründe geben, beispielsweise, weil es die Erben entnommen hatten, selbst behalten wollten oder es gesondert verkauft haben. Aber dass Pochmann dieses berühmte Selbstbildnis von Anton van Dyck nicht gekannt haben sollte, ist schwer vorstellbar.

Allerdings gab es auch Bildnisse anderer Künstler, die das Motiv der abgewinkelten Hand zei-
4 Vgl. Susan J. Barnes, Nora de Poorter, Oliver Millar, Horst Vey: Van Dyck. A Complete Catalogue of the Paintings, Yale 2004, Nr. I. 86, I. 87, S. 86, 87.

5 Vgl. ebd., Nr. I. 120, S. 114. Das Bild wird heute Van Dyck zugeschrieben: Dresden, Gemäldegalerie Alte Meister, Gal.-Nr. 960.

6 Vgl. Barnes u. a.: Van Dyck (wie Anm. 5), Nr. IV. 53, S. 471, 472.

7 Vgl. ebd., Nr. IV. 221, S. 602 603. - Man vergleiche auch das Porträt Karls I. im Ornat des Hosenbandordens im Schloss Windsor; Gustav Glück: Van Dyck. Des Meisters Gemälde. Mit 571 Abbildungen, New York 1931, S. 382.

8 Vgl. Barnes u. a.: Van Dyck (wie Anm. 5), Nr. II. 26, S. 169, 170,171

9 Auch bei späteren holländischen Malern findet sich diese Handhaltung, deutlich sichtbar beispielsweise auf dem Bildnis des Dionijs Wijnands (16281673), das Jan van Noordt im Jahre 1664 gemalt hat und das sich im Rijksmuseum in Amsterdam befindet (inv. no. A 709); vgl. David A. de Witt, Jan van Noordt: Painter of History and Portraits in Amsterdam, London, Ithaca 2007, S. 186, Catalogue 52.

$10 \mathrm{Vgl}$. auch das Bildnis eines Edelmannes mit Degen von Anton van Dyck im Louvre in Paris (Inv. 1248), das früher als Porträt des Malers Paul de Voss (1595-1678) gegolten hat und das um 1620 entstanden sein dürfte; vgl. Jacques Foucart: Catalogue des peintures flamandes et hollandaises du musée du Louvre, Paris 2009, S. 132.

11 Neuer Nekrolog (wie Anm. 1), S. 360-371.

12 Catalog der gewählten Kupferstichsammlung des Herrn Moritz Steinla [...] und des Nachlasses des Herrn T. L. Pochmann, Professor an der Königl. Sächs. Malerakademie zu Dresden, welche [...] öffentlich versteigert werden, Leipzig 1857. 
Jan van der Bruggen (geboren 1648 oder 1649) nach Anton van Dyck (1599-1641):

Selbstbildnis des Anton van Dyck, Schabkunst, 1682, $290 \times 224 \mathrm{~mm}$ (Platte), $295 \times 230 \mathrm{~mm}$ (Blatt) Staatliche Kunstsammlung Dresden, Kupferstich-Kabinett, Inv.-Nr.: A 32746

13 Tours, Musée des Beaux-Arts Richelieu, musée municipal Azay-le-Ferron, chateau, Tableaux français et italiens du $\mathrm{XVII}^{\mathrm{e}}$ siècle. Direction des musées de France. Inventaires des collections publiques françaises, 27 (Bearbeitet von Robert Fohr), Paris 1982, S. $45-$ 47, Nr. 34 (Abb. S. 46)

14 Musée National du Château de Versailles. Catalogue. Les Peintures. volumes II. Par Claire Constans, Paris 1995, Nr. 5755 (MV 3515), S. 1027.

15 Köln, Wallraf-Richartz-Museum, Katalog der Niederländischen Gemälde von 1550 bis 1800 im Wallraf-Richartz-Museum und im öffentlichen Besitz der Stadt Köln mit Ausnahme des Kölnischen Stadtmuseums, von Horst Vey, Annamaria Kesting, Köln 1967, S. 50, 51, Nr. 2529 .

16 Köln, Wallraf-Richartz-Museum, Katalog der Italienischen, Französischen und Spanischen Gemälde bis 1800, Köln 1973, S. 51-53, Nr. 1075

17 C. H. Collins Baker, W. G. Constable: Die Malerei des sechzehnten und siebzehnten Jahrhunderts in England, Berlin 1930, Tafel Nr, 39: das Bildnis des Lionel Fanshave von J. Michael Wright.

18 Ebd., Tafel Nr. 64. - Man vergleiche auch das Bildnis von Wright, A Highland Chief, in Edinburgh, Scottish National Portrait Gallery.

19 Vgl. Ellis Waterhouse: Painting in Britain 1530 to 1790 , Melbourne, London, Baltimore 1953, Abb. S. 56

20 Wien, Palais Dorotheum, Auktionskatalog 9. April 2014, Alte Meister, II. Teil, S. 157, lot 837; vgl. F. Petrucci: Ferdinand Voet (1639-1689) detto Ferdinando de' Ritratti, Roma 2005, S. 144, 145, Nr. 22a, 22b

21 Vgl. Harold Newman: An Illustrated Dictionary of Jewelry. Thames and Hudson, London 1981, S. 38 .

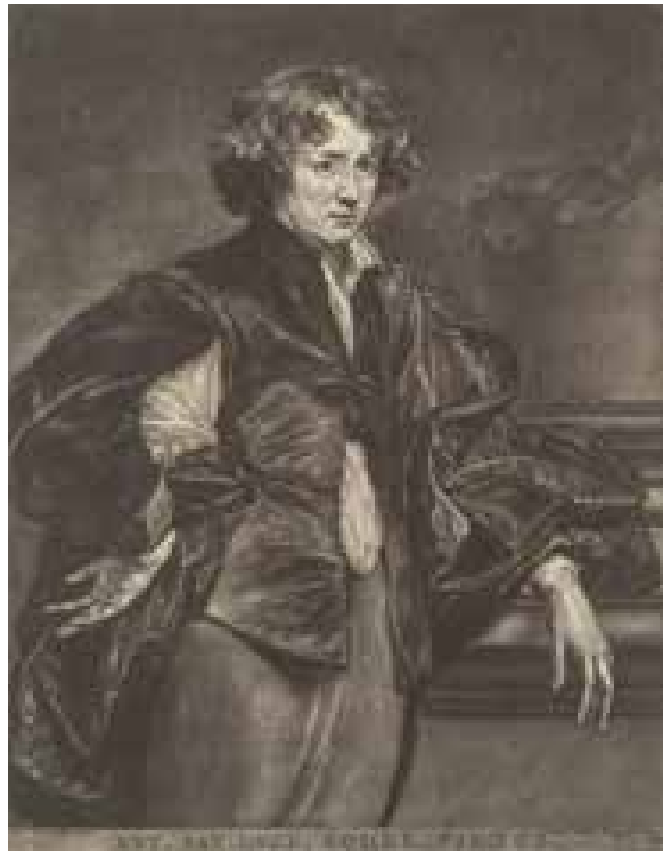

gen. Beispielsweise bewahrt in Tours das Musée des Beaux-Arts Richelieu, Musée Municipal Azay-le-Ferron, ein Reiterbildnis des Herzogs von Richelieu (1629-1715), das Charles de La Fosse (1636-1716) zugeschrieben wird; es zeigt den Herzog hoch zu Ross und könnte von Pierre Mignards Reiterbildnis König Ludwigs XIV. inspiriert sein. ${ }^{13}$ Lässig und elegant hat der Dargestellte die rechte Hand in die Hüfte gestützt. Armand Jean de Vignerot du Plessis, duc de Richelieu war ein Neffe des Kardinals Richelieu und großer Kunstsammler. Im Jahre 1665 verkaufte er Hauptwerke der Malerei aus seiner Sammlung an König Ludwig XIV.; diese Bilder befinden sich heute im Louvre.

Erwähnt werden kann auch das lebensgroße Porträt in ganzer Figur, das ein unbekannter französischer Maler von Henri de la Tour d'Auvergne, Vicomte de Turenne (1611-1675) geschaffen hat, als Allegorie auf dessen Siege im Jahre 1644; der nach links schreitende Vicomte de Turenne hat die linke Hand mit nachlässiger Gebärde abgewinkelt in die Hüfte gestützt. ${ }^{14}$

Auf einem Pastell-Porträt des Louis de France, Duc de Bourgogne (1682-1712), geschaffen von Joseph Vivien (1657-1734), das sich im Louvre in Paris befindet, hält der Dargestellte in der abgewinkelten rechten Hand, geradezu hinter seinem Rücken, die Geste motivierend, den Feldherrenstab; ein Kopie befindet sich im Château de Versailles (MV 3619).

Doch unzählige weitere Beispiele ließen sich anführen, so Bildnisse von König Ludwig XIV. und selbst Werke, die schon um 1600 entstanden sein dürften; hier wird nur auf einige besonders deutliche Gemälde dieser Art hingewiesen. So besitzt das Wallraf-Richartz-Museum in Köln zwei Bildnisse, die sich in ähnlicher Art mit der angewinkelten und verdrehten Hand auseinandersetzen. Das eine ist das Bildnis eines Mannes von Frans Hals ${ }^{15}$, das andere das Porträt eines unbekannten Malers von Vittore (Giuseppe) Ghislandi, auch Fra Paolotto und Fra Galgario genannt (1655-1743). ${ }^{16}$

Nennen wir schließlich noch das Porträt des James, erster Herzog von Hamilton, das Daniel Mytens d. Ä. (1599-vor 1648) im Jahre 1629 geschaffen hat ${ }^{17}$ sowie das Bildnis des Lionel Fanshave von J. Michael Wright. ${ }^{18}$ In verblüffender Weise hat auch Sir Peter Lely (16181680) die abgewinkelte Handhaltung von Van Dycks Selbstbildnis übernommen bei dem Gruppenbildnis der Familie des Charles Dormer, Earl of Carnarvon, gemalt um 1658/59. ${ }^{19}$ Ein besonderer Fall ist das Bildnis der jungen Christine von Schweden mit einem Löwen, gemalt in der Werkstatt des Ferdinand Voet (1639-1689), das bei der Versteigerung am 9. April 2014 im Dorotheum in Wien zum Aufruf gekommen ist. ${ }^{20}$ Es zeigt die Dargestellte in verblüffender Situation und Haltung: Sie schreitet anscheinend unbeirrt nach rechts, in einer Landschaft, hinter sich links eine kannelierte Säule, weiter hinten dicht belaubte Baumkronen. Furchtlos legt sie einem Löwen die linke Hand auf den gewaltigen Schädel mit den deutlich sichtbaren Zähnen - und die Rechte hat sie in der beschriebenen Weise in die Hüfte gestemmt.

Aber in dieser halb geöffneten rechten Hand hält sie einen durch die Arbeit des Gold- oder Silberschmiedes kugelig gefassten Bezoar als Schutz vor Vergiftung. ${ }^{21}$ Die Geste wirkt hier wie ein sichtbar gemachtes Geheimnis, wie der Hinweis darauf, dass die Dargestellte auf der Hut war, um ihr Leben zu schützen - und dass sie gleichzeitig mit der Kraft eines Löwen allen Angriffen trotzen wollte.

Es sind jedoch, wie wir bereits an dem Kölner Beispiel gesehen haben, nicht nur Könige, Fürsten und Adlige, sondern auch Künstler schon im 17. Jahrhundert mit der abgewinkelten Hand dargestellt worden. Die Haltung galt als besonders vornehm; so zeigte Jean Tiger (1623-1698) den Maler Nicolas Loir (16241670) auf einem Bildnis, das er 1675 als Rezeptions-Bild für die Königliche Akademie in Paris eingereicht hat, mit eben dieser Handhaltung.

Auch Pochmann ist diesem Darstellungs-Schema mehrfach gefolgt, so bei dem um 1805 entstandenen Porträt des Schauspielers Joseph Anton Christ. ${ }^{22}$ Der Dargestellte sitzt an einem Tisch, auf dem drei Bücher liegen und auf den er den linken Ellenbogen stützt, während die Hand mit dem ausgestreckten Zeigefinger die Schläfe und mit den drei anderen Fingern die Wange berührt. Die rechte Hand ist nach hinten abgewinkelt und nahe beim Körper auf den rechten Oberschenkel gestützt: eine wenig 


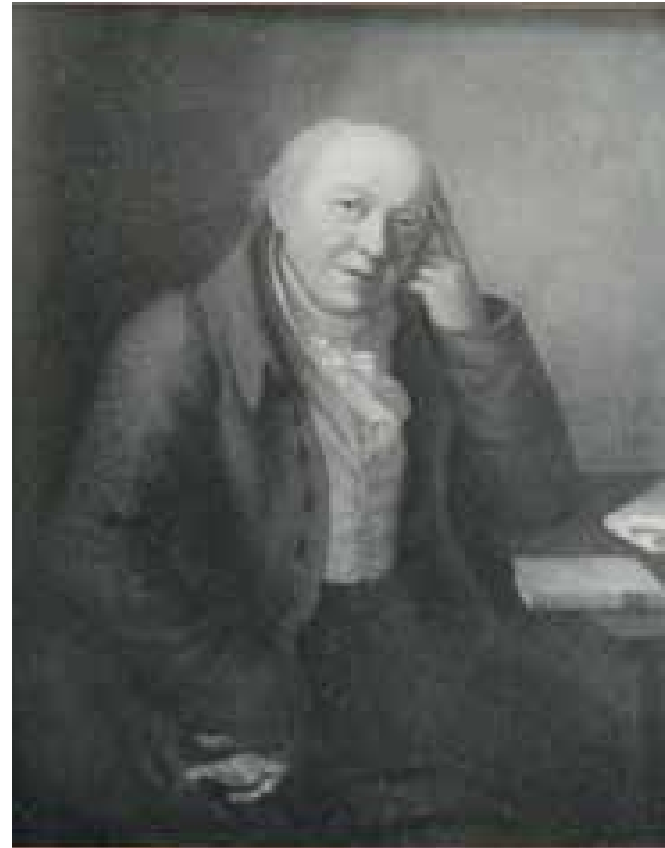

überzeugende Geste, die anscheinend dazu beitragen soll, den Eindruck nachdenklicher und doch entspannter Ruhe hervorzurufen.

Joseph Anton Christ (1744-1823) stammte aus Wien; er war ein bedeutender Charakterdarsteller. Wechselnde Engagements führten ihn u. a. nach Braunschweig, Leipzig und Berlin, nach Hamburg, St. Petersburg und Riga, nach Mainz, Prag und Dresden. Er war vermählt mit Isabella Maria Peixoto de Costa (aus Lissabon), zum zweiten Mal mit Fräulein Michelson und zum dritten Mal mit Johanne Amalie Karoline Schröder aus Freiberg, die er 1796 in Leipzig geheiratet hat.

Die auf seinem Porträt bemerkte, besondere Haltung der auf den Oberschenkel gestützten, abgewinkelten Hand finden wir auch bei dem Bildnis von Christs Schwiegersohn J. G. Bergmann. ${ }^{23}$ Der jugendlich und verträumt wirkende Dargestellte, ein seinerzeit und bis in die 1820er Jahre in Dresden und Leipzig gefeierter Schauspieler und Sänger, sitzt seitlich nach rechts gewendet vor einer weiten Landschaft und dreht sich dem Betrachter zu, den er anschaut. Man könnte ihn für einen romantischen Dichter halten, würde man seinen Beruf nicht kennen. Die rechte, nach hinten abgewinkelte Hand stützt er nahe am Körper auf den rechten Oberschenkel, während die linke auf dem hoch gestellten linken Knie ruht und ein Buch hält. Der Daumen ist zwischen die Seiten gelegt, so, als hätte der Leser nur für einen Augenblick die Lektüre unterbrochen, um sich dem Betrachter zuzuwenden. Andererseits wirkt der ernsthafte Blick in sich gekehrt und es ist, als würden wir den Porträtierten beim Rollenstudium beobachten. Rock, Weste und Halstuch sind - wie immer bei Pochmanns Männerporträts - der Zeit entsprechend und durchaus vornehm.

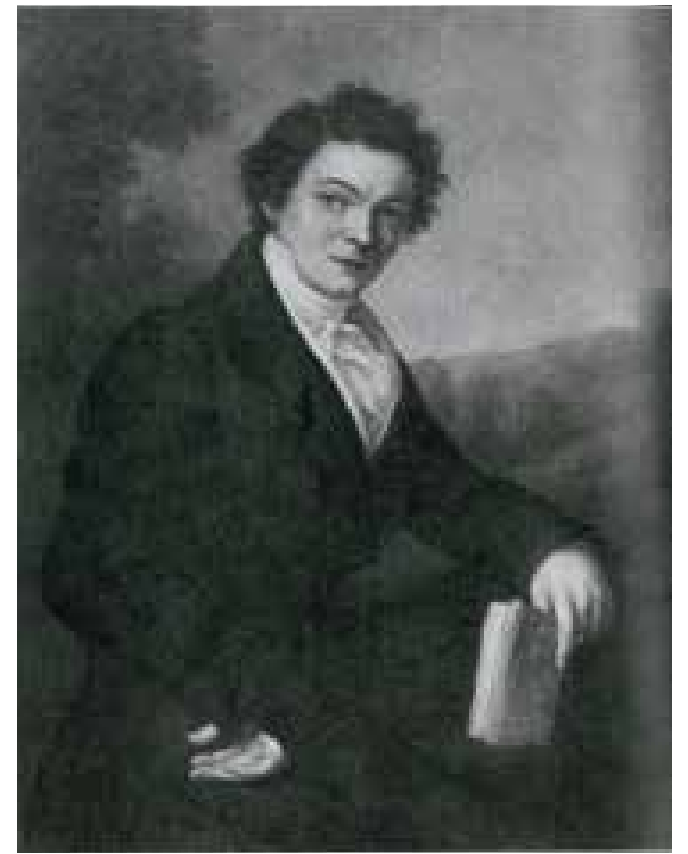

Pochmann hat auch Henriette Christ porträtiert, die Frau von J. G. Bergmann, also die Tochter von Joseph Anton Christ. Die beiden Bildnisse von Henriette und J. G. Bergmann nehmen aufeinander Bezug, wenden sich einander zu. Im Rücken der Dargestellten sind jeweils hohe Bäume angeordnet, vor ihnen ist die Landschaft offen. So sind sie als Gegenstücke erkennbar. Die Datierung stützt sich auf das etwaige Alter der Dargestellten und auf diesen Zusammenhang.

Schließlich soll noch darauf hingewiesen werden, dass dieses Motiv der abgewinkelten Hand schon in der Antike verwendet worden ist, so beim „Ausruhenden Satyr“ des Praxiteles, von dem sich eine Replik im Kapitolinischen Museum in Rom, eine andere in der Glyptothek in München erhalten hat. ${ }^{24}$ Ein Abguss des Exemplars aus dem Kapitolinischen Museum befindet sich in der Dresdner Abguss-Sammlung. ${ }^{25}$ Doch eindringlicher begegnet die beschriebene, von vielen Malern unterschiedlicher Schulen und Zeiten aufgegriffene Haltung der Hand bei dem erwähnten Selbstbildnis des von Pochmann hoch verehrten Anton van Dyck; er wird sein Vorbild gewesen sein.

Fragt man nach dem Ausdrucksgehalt der beschriebenen Geste, dann stellt man mit Erstaunen fest, dass ganz unterschiedliche Wirkungen mit der abgewinkelten, in die Hüfte gestützten Hand haben erzielt werden können, vom Eindruck der Furchtlosigkeit und Glaubenssicherheit, auch in historischen Szenen, bis hin zur überlegenen und demonstrativ zur Schau gestellten, manchmal sogar arrogant wirkenden Selbstsicherheit in Bildnissen; und immer konnte der Maler mit der Darstellung dieser komplizierten Handhaltung seine Virtuosität beweisen. Auch Pochmann hat es versucht. links: Traugott Leberecht Pochmann: Bildnis des

Schauspielers Joseph Anton Christ, um 1805, Öl auf Leinwand, Maße unbekannt, 1912 im Besitz von Rudolf Schirmer, gegenwärtiger Aufbewahrungsort unbekannt

rechts: Traugott Leberecht Pochmann: Bildnis des Sängers J. G. Bergmann, um 1805, Öl auf Leinwand, Maße unbekannt, 1912 im Besitz von Rudolf Schirmer, gegenwärtiger Aufbewahrungsort unbekannt
22 Vgl. Rudolf Schirmer: Schauspielerleben im achtzehnten Jahrhundert, Erinnerungen von Joseph Anton Christ, Ebenhausen-München, Leipzig 1912 , Abbildung nach S. 304. - Ernst Sigismund: Art. Traugott Leberecht Pochmann, in: Allgemeines Lexikon der bildenden Künstler, von der Antike bis zur Gegenwart. Begründet von Ulrich Thieme, Felix Bekker, hrsg. Hans Vollmer, Bd. 27 , Leipzig 1933, S. 169. - Harald Marx: „Hier fand er so viel Beschäftigung $[\ldots]$ “. Traugott Leberecht Pochmann als Porträtist in Leipzig, in: Sächsische Heimatblätter 58 ( 2012) 2. Heft, S. 114-135.

23 Vgl. Schirmer, Schauspielerleben (wie Anm. 24), Abbildung vor S. 289. - Sigismund: Art. Pochmann (wie Anm. 24), S. 169; Marx, Hier fand (wie Anm. 24).

24 Vgl. Peter C. Bol (Hrsg): Die Geschichte der antiken Bildhauerkunst. II. Klassische Plastik. Schriften des Liebighauses, Museum alter Plastik, Frankfurt am Main, Mainz 2004, Abb. 294, 295 a-f.

25 Staatliche Kunstsammlungen Dresden. Bildwerke des Altertums in Abgüssen. Aus dem Albertinum zu Dresden, Dresden 1953, S. 104, Nr. 168

\section{Autor}

Prof. Dr. Harald Marx

Dresden 